Yvonne Völkl

Spectatoriale Geschlechterkonstruktionen

Gender Studies 
Yvonne Völkl ist Literatur- und Kulturwissenschaftlerin im Fachgebiet der Romanistik. In ihrer Forschung beschäftigt sie sich u.a. mit Literatur und Presse des 18. Jahrhunderts, franko-kanadischer Migrationsliteratur sowie zeitgenössischen Corona Fictions. 
Yvonne Völkl

\section{Spectatoriale Geschlechterkonstruktionen}

Geschlechtsspezifische Wissens- und Welterzeugung in den französisch-

und spanischsprachigen Moralischen Wochenschriften des 18. Jahrhunderts

[transcript] 


\section{Bibliografische Information der Deutschen Nationalbibliothek}

Die Deutsche Nationalbibliothek verzeichnet diese Publikation in der Deutschen Nationalbibliografie; detaillierte bibliografische Daten sind im Internet über http://dnb.dnb.de abrufbar.

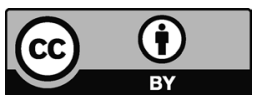

Dieses Werk ist lizenziert unter der Creative Commons Attribution 4.0 Lizenz (BY). Diese Lizenz erlaubt unter Voraussetzung der Namensnennung des Urhebers die Bearbeitung, Vervielfältigung und Verbreitung des Materials in jedem Format oder Medium für beliebige Zwecke, auch kommerziell. (Lizenztext:

https://creativecommons.org/licenses/by/4.o/deed.de)

Die Bedingungen der Creative-Commons-Lizenz gelten nur für Originalmaterial. Die Wiederverwendung von Material aus anderen Quellen (gekennzeichnet mit Quellenangabe) wie z.B. Schaubilder, Abbildungen, Fotos und Textauszüge erfordert ggf. weitere Nutzungsgenehmigungen durch den jeweiligen Rechteinhaber.

\section{Erschienen 2022 im transcript Verlag, Bielefeld}

\section{(c) Yvonne Völkl}

Umschlaggestaltung: Maria Arndt, Bielefeld

Umschlagabbildung: Yvonne Völkl

Lektorat: Gerrit Bayer-Hohenwarter

Druck: Majuskel Medienproduktion GmbH, Wetzlar

Print-ISBN 978-3-8376-6103-3

PDF-ISBN 978-3-8394-6103-7

https://doi.org/10.14361/9783839461037

Buchreihen-ISSN: 2625-0128

Buchreihen-eISSN: 2703-0482

Gedruckt auf alterungsbeständigem Papier mit chlorfrei gebleichtem Zellstoff.

Besuchen Sie uns im Internet: https://www.transcript-verlag.de

Unsere aktuelle Vorschau finden Sie unter www.transcript-verlag.de/vorschau-download 
„Wäre der Platz der Frauen in der jeweiligen Gesellschaft so ,natürlich“ vorgegeben, bedürfte es nicht aufwendiger Diskurse, ihn immer wieder zu bestimmen." Wunder (1988, 176)

„Narratives in general are not only one of the most powerful ways of worldmaking, but also of , self-making. "The main reason for this is that storytelling can generate real and possible worlds; narratives also exert performative power, i. e. they do not merely represent life, but they constitute and indeed ,form' life."

Nünning/Nünning $(2010,12)$

„Un Spectateur est une espèce de soleil (si l'on me passe cette comparaison), dont les rayons doivent éclairer tour à tour toutes les parties de la terre [...].“ Bastide (1759, NS2 IV, 16, 275) 
